\title{
5th biennial congress of the European Society of Endocrine Surgeons (ESES) Gothenburg Sweden, May 24-26, 2012
}

\section{Local Organizers:}

Bo Wängberg MD, PhD Gothenburg, Sweden

e-mail: bo.wangberg@surgery.gu.se

Svante Jansson MD, PhD, Gotheburg, Sweden

e-mail: svante.jansson@vgregion.se

\section{Scientific Committee:}

The Excutive committee of the ESES (see attached pdf file)

\section{OFFICERS}

Executive Council (2010 - 2012)

Jean-Francoise Henry, MD FRANCE jean-francois.henry@ ap-hm.fr President

Henning Dralle, MD GERMANY henning.dralle@medizin. uni-halle.de Past President

Bruno Niederle, MD AUSTRIA bruno.niederle@meduniwien. ac.at President Elect

Jean-Louis Kraimps, MD FRANCE j.l.kraimps@chu-poitiers.fr Secretary

Frederic Triponez, MD SWITZERLAND frederic.triponez@ hcuge.ch Treasurer

Anders Bergenfelz, MD SWEDEN anders.bergenfelz@med.lu.se Recorder

Rumen Pandev, MD BULGARIA rumenpandev@yahoo.com Chairman National Delegate Council

Antonio Sitges-Serra, MD SPAIN asitges@parcdesalutmar.cat DES- Representative

David Scott-Coombes, MD UNITED KINGDOM dscottcoombes@hotmail.com

Maurizio Iacobone, MD ITALY maurizio.iacobone@unipd.it

\section{RESEARCH and EDUCATION COMMITTEE} (2010 - 2012)

David Scott-Coombes, MD UNITED KINGDOM dscottcoombes@hotmail.com Chairman

Marcin Barczynski, MD POLAND marbar@mp.pl

Thomas Clerici, MD SWITZERLAND thomas.clerici@kssg.ch Kerstin Lorenz, MD GERMANY kerstin.lorenz@medizin. uni-halle.de

\section{NOMINATION COMMITEE (2010 - 2012)}

Henning Dralle, MD GERMANY henning.dralle@medizin. uni-halle.de Chairman

Dimitrios Linos, MD GREECE dlinos@hms.harvard.edu Celestino Lombardi, MD ITALY cp.lombardi@rm.unicatt.it Greg Sadler, MD UNITED KINGDOM gregsadler@ btinternet.com

\section{AUDITORS (2010-2012)}

Bertil Hamberger, MD SWEDEN bertil.hamberger@kirurgi.ki.se Marco Raffaelli, MD ITALY marcoraffaelli@rm.unicatt.it

\section{ESES VIENNA OFFICE}

Karin KNOB VIENNA kknob@medacad.org

NATIONAL DELEGATE COUNCIL (2010 - 2012)

Rumen Pandev, MD BULGARIA rumenpandev@yahoo.com Chairman

Christian Scheuba, MD AUSTRIA christian.scheuba@ meduniwien.ac.at

Rumen Pandev BULGARIA rumenpandev@yahoo.com

Sam Van Slycke BELGIUM Sam.Van.Slycke@olvz-aalst.be Danijel Dosen CROATIA danijel.dosen@kzt.hr Peer Christiansen DENMARK peerchri@rm.dk Bruno Carnaille FRANCE bcarnaille@chru-lille.fr Dietmar Simon GERMANY dsimon@online.de Dimitrios Linos GREECE dlinos@hms.harvard.edu Celestino Pio Lombardi ITALY cp.lombardi@rm.unicatt.it Zenons Narbuts LATVIA narbuts@apollo.lv Virgilijus Beisa LITHUANIA virgilijus.beisa@santa.lt Jan Erik Varhaug NORWAY jan.erik.varhaug@kir.uib.no Stanislaw Cichon POLAND stanislaw.cichon@interia.pl Jose Emilio Craveiro Rocha PORTUGAL jec.rocha@sapo.pt Aleksandrau Grigiorovici ROMANIA 1xndrgrgrvc8@gmail.com Ivan Paunovic SERBIA Prof.Paunovic55@gmail.com Nikola Besic SLOVENIA nbesic@onko-i.si Pablo Moreno SPAIN 25108pml@gmail.com Claes Juhlin SWEDEN claes.juhlin@akademiska.se Christof Kull SWITZERLAND christof.kull@ksli.ch 
Serdar Tezelman TURKEY serdar.tezelman@gmail.com Greg Sadler UNITED KINGDOM gregsadler@btinternet.com Sergiy Cherenko UKRAINE endosurg@unet.net.ua

\section{HONORARY MEMBERS}

N. W. Thompson, MD USA 2006 - Krakow

M. H. Wheeler, MD UNITED KINGDOM 2006 - Krakow

M. Rothmund, MD GERMANY 2008 - Barcelona

A. E. Young, MD UNITED KINGDOM 2010 - Vienna

\section{Oral Presentations:}

Topic: Thyroid

Presentation type: Oral presentation

Ozer Makay ${ }^{1}$, Pinar Karakan ${ }^{1}$, Selen Bayraktaroglu ${ }^{1}$, Gökhan Icoz ${ }^{1}$, Naim Ceylan ${ }^{1}$, Mahir Akyildiz ${ }^{1}$, Hudaver Alper $^{1}$

${ }^{1}$ Ege University, Turkey

\section{3-Tesla apparent diffusion coefficient values in differentiating thyroid nodules: preliminary report}

Purpose: The aim of this study was to assess the diagnostic value of 3-T diffusion-weighted imaging for the characterization of thyroid nodules.

Methods: This initial report included 15 prospectively investigated thyroidectomized patients, where 3-T diffusion-weighted imaging had been performed prior to surgery. A total of 35 nodules were assessed with the $b$ factors 500 and $1000 \mathrm{~mm} / \mathrm{s}$, by using single-shot echoplanar imaging. Apparent diffusion coefficient (ADC) values of thyroid nodules in subjects were calculated and correlated with the postoperative histopathological results. The Mann-Whitney $U$ test was used for statistical analysis.

Results: Histologically, there were 7 carcinomas with a minimum size of $1,3 \mathrm{~cm}$. The mean ADC values differed significantly among malignant and benign nodules for both $\mathrm{b}$ factors $(\mathrm{p}$

Conclusion: 3-Tesla ADC values show potential for differentiating malignant nodules from benign ones. Although it seems feasible, more patients are required for confirmation of our initial results.

E-mail presenting author: ozer.makay@ege.edu.tr

\section{Topic: Thyroid}

Presentation type: Oral presentation

Carmen Gonzalez-Sanchez ${ }^{1}$, Guzman Franch-Arcas ${ }^{1}$, Paloma Garcia-Talavera ${ }^{2}$, Angel Muñoz-Herrera ${ }^{1}$, Nicolas Macias ${ }^{1}$, Ana Sanchez ${ }^{1}$, Orlando Rozo ${ }^{1}$, Yary Aguilera ${ }^{1}$, Alberto Gomez-Alonso ${ }^{1}$

${ }^{1}$ Hospital Universitario Salamanca

${ }^{2}$ Hospital Clinico Valladolid, Spain
Morbidity following thyroid surgery: does surgical volume matter?

Purpose: The aim was to evaluate the surgical volume and morbidity of patients operated on by endocrine surgery dedicated surgeons (group EndS) compared with general department surgeons (group GenS) in a tertiary institution. Methods: We present the results of a prospective cohort study of all patients submitted to thyroid surgery in our Institution (between January 2008 and August 2010). They have been followed for 12 months at least. Postoperative recurrent nerve function was assessed by laryngoscopy. Calcaemia was monitored in all patients until achieving normality with no need for vitamin $\mathrm{D}$ and oral calcium supplementation.

Results: We studied 225 patients, 195 in group EndS (2 surgeons with $>40$ procedures per surgeon and year) and 30 in group GenS (6 surgeons with $<5$ procedures). Number of exposed nerves was 325 and 46 respectively. Prevalence of recurrent nerve palsy persisting beyond 12 months was $1 / 325$ and 2/46 of exposed nerves $(p=0.04)$. Prevalence of persisting hypocalcaemia beyond 12 months was $3 / 195$ and $3 / 30$ of patients $(p=0.03)$.

Conclusion: 1. Morbidity in terms of permanent recurrent nerve palsy and hypocalcaemia was less frequent among patients operated by endocrine surgeons.

2. Differences in surgical volume may explain these variations in morbidity.

E-mail presenting author: crmngs@hotmail.com

Topic: Thyroid

Presentation type: Oral presentation

Joan J. Sancho ${ }^{1}$, Rosa Prieto $^{2}$, Joaquín Ripollés ${ }^{3}$, Carles Ribera $^{3}$, Juan Sánchez ${ }^{2}$, Antonio Sitges-Serra ${ }^{2}$

${ }^{1}$ Hospital del Mar

${ }^{2} \mathrm{H}$. del Mar

${ }^{3}$ H. de Martorell, Spain

\section{A randomised trial of hemithyroidectomy vs. Dunhill for asymmetrical goiter}

Purpose: Half of the patients presenting with an unilateral benign thyroid nodule have contralateral subclinical disease. There is controversy whether these patients should be treated with hemithyroidectomy or with a bilateral procedure.

Methods: Adult patients (18-65 yrs.) with a benign unilateral dominant nodule and contralateral nodule(s) $<10 \mathrm{~mm}$. detected on neck US were randomized to hemithyroidectomy (HMT) or Dunhill-Near total thyroidectomy (DNT). Rates of complications, remnant growth, incidental carcinoma and reoperation were assessed.

Results: One-hundred eighteen patients (F/M:118/8, mean age 43 yrs.) were initially included and randomized: 65 to HMT and 53 to DNT. After randomization 28 patients were excluded 
leaving 47 HMT and 43 DNT long-term ( $55 \pm 35$ months) evaluable patients. Mean nodule size was 38 and $6 \mathrm{~mm}$ for the dominant and contralateral nodules respectively. No differences were found in operative time, accidental parathyroidectomy, parathyroid autotransplantation, histopathology or wound complications. Transient hypocalcemia (s-Ca $<8 \mathrm{mg}$ at $24 \mathrm{~h}$ ) was more common in DNT (30 vs. $8 \% ; P<0.001)$. No permanent complications were observed in either group. $30 \%$ of HMTs required T4 supplementation. At the last followup visit, [TSH] was the same in both groups. Remnant growth ( 20 vs $0 \% ; P<.001)$, appearance of new nodules ( 55 vs $14 \% ; P$ $<0.001)$ and overall reoperation rate as per intention to treat ( 9.2 vs $1.8 \%, P=0.2$ ) were more common in HMT.

Conclusions: DNT appears superior to HMT in terms of reoperation and progression/recurrence rates and has a similarly uneventful immediate postoperative course.

E-mail presenting author: asitges@hospitaldelmar.cat

Topic: Parathyroid

Presentation type: Oral presentation

Ming $\mathrm{Lu}^{1}$, Lars-Ove Farnebo ${ }^{1}$, Rober Bränström ${ }^{1}$, Catharina Larsson $^{1}$

${ }^{1}$ Karolinska Institutet, Sweden

\section{Regulation of parathyroid hormone secretion by caffeine-Potential impact on osteoporosis}

Purpose: Independent studies have reported associations between caffeine consumption, low bone mineral density, as well as impaired bone development in vitro and in vivo. Besides calcium $(\mathrm{Ca} 2+)$ and vitamin $\mathrm{D}$, secretion of parathyroid hormone (PTH) is known as a critical regulator of bone remodeling. The effect of caffeine on parathyroid hormone secretion in parathyroid cell has never been explored.

Methods: Using short term cultured parathyroid cells from patients with primary hyperparathyroidism (PHPT), we assessed the effects of caffeine on PTH secretion, intracellular $\mathrm{Ca} 2+[\mathrm{Ca} 2+] \mathrm{i}$ and cAMP.

Results: We showed that $200 \mu \mathrm{M}$ and higher doses of caffeine inhibited intact PTH secretion in human parathyroid adenoma cells using perifusion technique. However, [Ca2+]i determined by Fura- 2 was not affected by caffeine at any of the doses ( 50 , $200,500 \mu \mathrm{M}$ or $5 \mathrm{mM}$ ). Two main caffeine targets, Adenosine receptors $\mathrm{A} 1$ and $\mathrm{A} 2 \mathrm{~A}$, were demonstrated in human normal parathyroid and parathyroid adenomas. Measurements of intracellular cAMP showed decreased cAMP at $50 \mu \mathrm{M}$ caffeine, and gradually increased cAMP at higher concentrations.

Conclusion: High doses of caffeine inhibit PTH secretion in human parathyroid cells independent of intracellular $\mathrm{Ca} 2+$ and cAMP level. The inhibition of PTH secretion caused by high doses of caffeine may contribute to bone loss in human.

E-mail presenting author: ming.lu.1@ki.se
Topic: Parathyroid

Presentation type: Oral presentation

Lars Rolighed $^{1}$, Peter Vestergaard ${ }^{2}$, Lene Heickendorff ${ }^{3}$, Tanja Sikjaer $^{2}$, Lars Rejnmark ${ }^{2}$, Leif Mosekilde ${ }^{2}$, Peer Christiansen ${ }^{4}$

${ }^{1}$ Aarhus University Hospital

${ }^{2}$ Dept. Endocrinology MEA

${ }^{3}$ Dept. Clin. Biochemistry,

${ }^{4}$ Dept. Surgery P, Denmark

\section{Bone mineral density improvements after operation for primary hyperparathyroidism.}

Purpose: In primary hyperparathyroidism one of the arguments for operation is decreased BMD in the spine, hip or forearm. However, it can be an option to observe patients in asymptomatic cases.

Methods: Analysis of a historic consecutive cohort of 236 PHPT patients where DXA scans pre- and 1 year postoperatively, clinical data, and biochemical data were available. Results: Mean age was 60 years (range 19-86) and $81 \%$ were women. A significant postoperative increase in spine BMD of $3.4 \%$ was seen. In a multiple regression model the BMD increase was positively associated with preoperative PTH but not with other clinical or biochemical variables. Hip BMD increased $2.1 \%$. The BMD increase was positively associated with preoperative PTH and negatively associated with creatinine and weight. Forearm BMD decreased by $-0.6 \%$ and the BMD change was positively associated with PTH. In 91 patients with mild PHPT ( $\mathrm{iCa}<1.45 \mathrm{mmol} / \mathrm{l})$ there were significant postoperative increases in BMD in both the spine $(2.6 \%)$ and hip (1.3\%), but a decrease in the forearm BMD $(-0.7 \%)$. Conclusions: Significant postoperative BMD improvements in both the hip and spine were seen. BMD improvements were also significant in mild cases. At all scan sites there was a positive association with PTH and BMD increase. This improvement may be due to reduced remodeling activity.

E-mail presenting author: larsrolighed@gmail.com

Topic: Adrenal

Presentation type: Oral presentation

Maurizio Iacobone ${ }^{1}$, Marilisa Citton $^{2}$, Giovanni Viel $^{2}$, Riccardo Boetto $^{2}$, Italo Bonadio ${ }^{2}$, Isabella Mondi ${ }^{2}$, Saveria Tropea $^{3}$, Donato $\mathrm{Nitti}^{4}$, Gennaro Favia ${ }^{2}$

${ }^{1}$ University of Padua

${ }^{2}$ Endocrine Surgery, University of Padua

${ }^{3}$ Endocrinology Unit, University of Padua

${ }^{4}$ Endocrine Surgery Unit, University of Pa, Italy

Outcomes of Adrenalectomy in adrenal incidentalomas with subclinical Cushing's syndrome

Purpose: Adrenalectomy represents the definitive treatment in clinically evident Cushing's Syndrome, while the most 
appropriate treatment in case of subclinical Cushing's Syndrome (SCS) in adrenal incidentalomas remains controversial. This study was aimed to compare the outcome of adrenalectomy or conservative management in adrenal incidentalomas with SCS. Methods: Twenty patients underwent laparoscopic adrenalectomy for SCS in adrenal incidentaloma, while 15 were managed conservatively. Hormonal laboratory parameters of corticosteroidal secretion, arterial blood pressure (BP), glycometabolic control parameters, and quality of life (by the SF-36 questionnaire) were assessed pre and postoperatively. Results: The 2 groups were equivalent concerning demographics, preoperative laboratory data, BP levels. In the surgical group, no postoperative morbidity occurred; laboratory corticosteroidal parameters normalized in all patients while never in the conservative group $(p<0.01)$. In operated patients, a normalization or significant amelioration of BP was achieved in 53\% and glucose intolerance recovered in 54\% of cases; while in the conservative group no significant ameliorations or some worsening occurred $(p<0.01)$. SF-36 physical and mental components significantly ameliorated in the surgical group $(p<0.01)$. Conclusions: Adrenalectomy can be more beneficial than conservative management in SCS. Surgery may achieve remission of laboratory hormonal abnormalities, improve $\mathrm{BP}$ values, glycemic control, and quality of life.

E-mail presenting author: maurizio.iacobone@unipd.it

Topic: Thyroid

Presentation type: Oral presentation

Kerstin Lorenz ${ }^{1}$, Malik Elwerr ${ }^{1}$, Henning Dralle ${ }^{1}$

${ }^{1}$ University of Halle-Wittenberg, Germany

The relevance of "false positive" calcitonin stimulation testing: Spectrum and surgical implication

Purpose: Routine calcitonin measurements should discriminate c-cell-hyperplasia $(\mathrm{CCH})$ from medullary thyroid carcinoma (MTC). Elevated calcitonin levels effect stimulation testing, determining indication and extent of surgery.

Methods: 50 patients underwent stimulated calcitonin determination with pentagastrin $(80 \%)$ or calcium $(20 \%)$ pre- and postoperatively. Indications to surgery were nodular goiter (BNG), suspected CCH or MTC, RET-genecarriership and suspected differentiated thyroid cancer. Prevalent extrinsic sources of hypercalcitonemia were excluded.

Results: Mean calciumstimulated calcitonin were 398 thyroiditis, 44 RET-carriers, 152 non-MTC thyroid carcinoma and 161 BNG, with Pentagastrin 78 thyroiditis, 32 RET-carriers, 122 non-MTC thyroid carcinoma, 96 BNG. All underwent total thyroidectomy, bilateral cervical central (9), lateral (1) lymphadenectomy. Histology showed 9 non-medullary carcinomas, 4 thyroiditis, 28 BNG. Immunhistochemistry excluded $\mathrm{CCH}$ and MTC. Except 1, all showed normalized basal and stimulated calcitonin postoperatively.
Conclusions: Calcium stimulation provokes higher calcitonin and increased response factors compared to pentagastrin. Thyroid pathology without $\mathrm{CCH}$ and $\mathrm{MTC}$ evidence sequentially associates with calcium stimulation: RETcarriers, BNG, thyroiditis, non-MTC thyroid carcinoma. Discriminating these, extrinsic sources, $\mathrm{CCH}$ and $\mathrm{MTC}$ determine indication and extent of surgery. Lacking defined cut-off values, ranges of basal calcitonin below $50 \mathrm{pg} / \mathrm{ml}$ and below 400 in calcium-stimulation revealed no c-cell disease in this series.

E-mail presenting author: kerstin.lorenz@uk-halle.de

Topic: Parathyroid

Presentation type: Oral presentation

Sergio Salido ${ }^{1}$, Joaquín Gómez ${ }^{2}$, Jose Luís Muñoz-De Nova ${ }^{2}$, Guillermo Fernández-Díaz ${ }^{2}$, Jose Miguel Bravo ${ }^{2}$, Maria Posada $^{2}$, Jorge Auza ${ }^{2}$, Elena Martín-Pérez ${ }^{2}$, Eduardo Larrañaga ${ }^{2}$ ${ }^{1}$ La Princesa Hospital

${ }^{2}$,Spain

\section{Normocalcemic primary hyperparathyroidism: bone} status, imaging localization and pathological features.

Purpose: from 1953 normocalcemic hyperparathyroidism has been described by many authors. But most of its peculiarities remain unknown.

Methods: A retrospective case-control study has been realized in our endocrine surgery department from January 2001 and November 2011. We compared 108 patients suspected to have a parathyroid adenoma with either normocalcemic or hypercalcemic status. Personal antecedents, biochemical registers, imaging, surgery, pathological features and rate of cure have been analyzed. Results: Bone loss before surgery was higher among normocalcemic patients $(46.3 \%$ vs. $24.1 \% ; p=0.023$ ) and pathological fractures $(25.9 \%$ vs. $11.1 \% ; p=0.048)$. Also alkaline phosphatase was increased in normocalcemic patients $(129 \mathrm{mg} / \mathrm{dl}$ vs. $95 \mathrm{mg} / \mathrm{dl} ; p=$ $0.001)$. Multiple gland resections were necessary in more normocalcemic patients ( $16 \%$ vs. $3.7 \%$; $p=0.001)$. Sensitivity ( $85.2 \%$ vs. $88.9 \%$ ) and positive predictive value (59.3 vs. $83.3 \%$ ) were inferior for normocalcemic cases with statistical significance $(p=0.001)$. Weight gland was lesser in normocalcemic $(0,44 \mathrm{~g}$ vs. $0,86 \mathrm{~g} ; p=0.03)$ even when 43 cases were matched by PTH levels ( $0.4 \mathrm{~g}$ vs. $0.77 ; p=0.014)$. Finally cure rate was different against normocalcemic $(62.7 \%$ vs. $95.7 \%, p<0.001)$, but not in case of using PTH levels as cure parameter after surgery $(66.7 \%$ vs. $68.1 \% ; p=0.831)$.

Discussion: There are many differences between both hyperparathyroidism conceptions, prospective studies are needed for corroborate those findings.

E-mail presenting author: salidosergio@hotmail.com 
Topic: Parathyroid

Presentation type: Oral presentation

Denis Wirowski ${ }^{1}$, Katharina Schwarz ${ }^{2}$, Bernhard J. Lammers $^{2}$, Hans-Dietrich Röher ${ }^{3}$, Peter E. Goretzki ${ }^{2}$

${ }^{1}$ Lukaskrankenhaus Neuss

${ }^{2}$ Dept. of Surgery, Lukaskrankenhaus

${ }^{3}$ Dept. of Surgery, Univ. Hosp. Düsseldorf, Germany

Failed surgery in PHPT-have improved pre- and intraoperative imaging or diagnostic tools changed the outcome?

Purpose: Advanced preoperative imaging of parathyroid adenomas and intraoperative parathyroid hormone determination optimized the results in the surgical treatment of PHPT patients. Nevertheless, surgery is not always successful. We asked, whether reasons for failure have changed during the last 25 years. Methods: We retrospectively analyzed operations for persistent PHPT in our department between 2001 and $2011(n=62)$ and compared these results to our experience between 1986 and $2001(n=81)$.

Results: Of 757 operations for PHPT between 2001 and 2011, 62 (8\%) were for persistent disease. Main reasons for failure were a misdiagnosed multiple gland disease $(n=$ $15 / 25,60 \%$ ) in our own group of patients, and an undetected solitary adenoma $(n=22 / 37,59 \%)$ in patients being operated primarily somewhere else. All patients with persistent PHPT were cured. Between 1986 and 2001, main indications for reoperation were an undiagnosed multiple gland disease $(15 / 24,63 \%)$ in our own patients, and a missed solitary adenoma (38/57, 67\%) in outward patients.

Conclusions: Comparing our experience in 143 patients with persistent PHPT being operated between 2001-2011 and 1986-2001, not much has changed, despite the new armamentarium of improved preoperative imaging or intraoperative biochemical control. These results support the referral of PHPT patients to experienced endocrine surgery units.

E-mail presenting author: denis.wirowski@t-online.de

Topic: Thyroid

Presentation type: Oral presentation

Radan Dzodic ${ }^{1}$, Ivan Markovic ${ }^{2}$, Igor Djurisic ${ }^{2}$, Marko Buta $^{2}$, Merima Oruci ${ }^{2}$, Marko Jevric ${ }^{2}$, Zorka Milovanovic ${ }^{2}$, Gordana Pupic ${ }^{2}$

${ }^{1}$ University of Belgrade, IORS

${ }^{2}$ Inst of Oncol and Rad of Serbia, Serbia

Sentinel lymph node biopsy in differentiated thyroid carcinoma and decision for selective modified radical neck dissection

Purpose: The accuracy of sentinel lymph node biopsy in decisions for surgical management of lymph nodes in differentiated thyroid ccarcinoma was demonstarted in few previous studies.

Methods: We have performed SLN biopsy in 172 patients with DTC. Before mobilization of the thyroid gland, $0.2 \mathrm{ml}$ of $1 \%$ solution of methylen blue dye was injected peritumorally. After 10 min the dissection was continued around omohyoid muscle, towards the internal jugular vein and carotid artery until blue stained LN were found and sent for frozen-section examination. An extended dissection of level III and IV was done consecutively. All LN were examined by frozen section and conventional histopathology examination. If positive, MRND was performed after total thyroidectomy and routine dissection of central neck compartment.

Results: Identification rate of SLN was $93.5 \%$. Specificity and sensitivity of the method were $100 \%$ and $80 \%$ respectively. Negative and positive predictive values were $94.7 \%$ and $100 \%$. Overall accuracy of the method was $95.6 \%$.

Conclusions: Our results imply that SLNb in the jugulocarotid chain using methylene blue dye mapping, is feasible and accurate method for estimating LN status in the lateral neck compartment. The method may support a decision to perform selective MRND in patients with DTC

E-mail presenting author: radan.dzodic@gmail.com

Topic: Parathyroid

Presentation type: Oral presentation

Haridimos Markogiannakis ${ }^{1}$, Nikolaos Memos ${ }^{1}$, Dimitrios Tsamis $^{1}$, Nikolaos V. Michalopoulos ${ }^{1}$, Ioannis Manouras ${ }^{1}$, Artemisia Papadima ${ }^{1}$, Emmanuel Lagoudianakis ${ }^{1}$, George C. Zografos ${ }^{1}$, Andreas Manouras ${ }^{1}$

${ }^{1}$ Hippokration Hospital, Athens University, Greece

\section{Evaluation of cystatin $C$ in patients operated for primary hyperparathyroidism}

Purpose: Serum cystatin $\mathrm{C}$ has been suggested as a new sensitive marker of renal function. Our objective was to evaluate preoperative cystatin $\mathrm{C}$ in patients operated for primary hyperparathyroidism and to identify factors associated with it.

Methods: All patients with primary hyperparathyroidism undergoing parathyroidectomy during a 3-year period were prospectively included. Parameters evaluated for association with cystatin $\mathrm{C}$ were: demographics, hyperparathyroidism symptoms, bone mineral density, parathyroid weight and diameter, preoperative $24 \mathrm{~h}$ urinary calcium, and preoperative serum creatinine, albumin, T3, T4, FT3, FT4, TSH, anti-Tg, anti-TPO, calcium, phosphate, PTH and 25(OH) VitD3. 


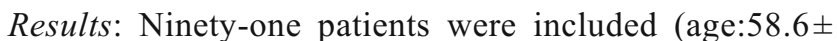
7.1 years "female:74.7\%). Elevated cystatin $\mathrm{C}$ was noticed in $47(51.6 \%)$ patients whereas creatinine in only $7(7.7 \%)$. Cystatin $\mathrm{C}$ had a positive association with preoperative PTH $(p=0.04)$, calcium $(p=0.04)$ and albumin $(p=0.01)$. In the multivariate analysis, cystatin $\mathrm{C}$ was correlated with PTH $(p=0.02)$ and albumin $(p=0.02)$.

Conclusion: Cystatin $\mathrm{C}$ is independently correlated with PTH and may therefore be elevated in primary hyperparathyroidism. This association is likely indicating renal impairment but other mechanisms may also be involved. Cystatin $\mathrm{C}$ seems to be an earlier and more accurate marker of renal function in primary hyperparathyroidism patients than other traditionally used parameters.

E-mail presenting author: markogiannakis@yahoo.com

Topic: Parathyroid

Presentation type: Oral presentation

Erik Nordenström ${ }^{1}$, Antonio Sitges Sierra ${ }^{2}$, Anders Bergenfelz ${ }^{3}$, Joan J Sancho ${ }^{2}$, Mark Thier ${ }^{3}$, Martin Almqvist ${ }^{3}$

${ }^{1}$ Lund University Hospital, Sweden

${ }^{2} 21$ Endocrine Surgery Unit, Department of, Spain

${ }^{3}$ Lund University, Sweden

\section{Vitamin D status and bone remineralization after surgery for primary hyperparathyroidism}

Purpose: It is not clear if postoperative gain in bone mineral density (BMD) after surgery for primary hyperparathyroidism (PHPT) is influenced by vitamin D status. The aim of this study was to investigate if PHPT patients from Spain and Sweden differed in vitamin D status, disease activity and bone remineralization after surgery.

Methods: This investigation was a prospective observational study of two cohorts of postmenopausal women from Spain $(n=126)$ and Sweden $(n=128)$ that had surgery for sporadic PHPT. Biochemical variables reflecting bone metabolism and disease activity including vitamin D and BMD was measured pre and 1 year postoperatively.

Results: 25(OH)D3 levels were lower in the Spanish patients and they had higher preoperative levels of PTH (13.5 vs $11.0 \mathrm{pmol} / \mathrm{L}, p<0.001), 1.25(\mathrm{OH}) 2 \mathrm{D} 3(142$ vs $56 \mathrm{pmol} / \mathrm{L}$, $p>0.001$ ), urinary calcium ( $7.3 \mathrm{vs} 4.1 \mathrm{mmol} / \mathrm{L}, p<0.001$ ) and heavier adenomas (620 vs $500 \mathrm{~g}, p<0.001)$.Increase in BMD 1 year after surgery was correlated to vitamin D levels, disease activity and nationality.

Conclusion: Postmenopasual women with PHPT from Spain have a more advanced disease and lower vitamin $25(\mathrm{OH}) \mathrm{D} 3$ levels. Improvement in bone density 1 year after surgery is correlated to disease severity and vitamin D levels.

E-mail presenting author: erik.nordenstrom@skane.se
Topic: Thyroid

Presentation type: Oral presentation

Nuria munoz perez ${ }^{1}$, Jesus villar del moral ${ }^{1}$, Maria angustias muros fuentes ${ }^{1}$, Juan ignacio arcelus martinez ${ }^{1}$, Andrea vilchez rabelo ${ }^{1}$, Natalia zambudio carrol $^{1}$, Patricia becerra massare $^{1}$, Martin lopez de la torre ${ }^{1}$, Jose antonio ferron orihuela $^{1}$

${ }^{1}$ Virgen de las nieves university hospital, Spain

\section{Could 18 F-FDG-PET/CT avoid unnecessary thyroidectomies in patients with cytological diagnosis of follicular neoplasm?}

Purpose: Preoperative diagnosis of thyroid nodules with "follicular neoplasm" in fine-needle aspiration biopsy (FNAB) forces thyroidectomy to exclude malignancy. This study evaluates the role of 18 F-fluorodeoxyglucose positron emission tomography/computed tomography (18 FFDG-PET/CT) to prevent unnecessary thyroidectomies in this clinical setting.

Methods: Prospective study of 42 patients scheduled for thyroidectomy due to follicular neoplasm cytology in FNAB (32 follicular, 10 Hürthle cell neoplasms, according to Bethesda classification) since May 2009. All patients underwent preoperative 18 F-FDG-PET/CT. Abnormal 18 F-FDG thyroid uptake was assessed visually and by measuring the maximum standardized uptake value (SUVmax). Results were compared with final pathology reports.

Results: 12 of 42 patients (28\%) were finally diagnosed as thyroid cancer. Focal uptake correlated with a greater risk of malignancy $(p=0.031) .18$ F-FDG-PET/CT uptake showed sensitivity, specificity, positive and negative predictive values and overall accuracy of $92 \%, 47 \%, 41 \%$, $93 \%$ and $60 \%$, respectively. The optimal threshold SUVmax to discriminate malignancy was 4.5 (area under ROC curve of 0.73 ). Use of 18 F-FDG-PET/CT could reduce by $47 \%$ the thyroidectomies performed for finally benign nodules.

Conclusions: 18 F-FDG-PET/CT can play a role in the management of nodules cytologically reported as "follicular neoplasm", allowing the avoidance of a significant number of thyroidectomies for finally benign lesions.

E-mail presenting author: numupe@ono.com

Topic: Thyroid

Presentation type: Oral presentation

Sam Van Slycke ${ }^{1}$, Sam Van Slycke ${ }^{1}$, Jean-Pierre Gillardin ${ }^{1}$, Nele Brusselaers ${ }^{2}$, Hubert Vermeersch ${ }^{2}$

${ }^{1}$ OLV Clinic Aalst

${ }^{2}$ University Hospital Ghent, Belgium 


\section{S-shaped electrode for continuous vagal nerve stimulation in thyroid surgery}

Purpose: Intra-operative neuromonitoring of the recurrent laryngeal nerve and vagal nerve are performed as an adjunct to the gold standard of optical visualisation of the RLN during thyroid surgery to prevent injury of the RLN, and subsequently paralysis of the vocal cords. Actually there are no data available about continuous vagal stimulation and it's value in predicting vocal cord injury.

Methods: A single institution prospective study was performed: 100 thyroidectomies with continuous vagal nerve stimulation were analysed. The monitoring has been performed with Avalanche $X T^{\circledR}$ from Dr. Langer.

Results: 180 nerves at risk were monitored with 4 temporary recurrent laryngeal nerve palsies, with a clear demonstration of diminution of the amplitude, recovering partially with release of traction. Changes in latency time were not significant. Loss of signal was obtained in 4 cases with localisation of the suspected area of injury by bipolar stimulation of the recurrent laryngeal nerve.

Conclusion: Continuous neuromonitoring of the vagal nerve can help in predict vocal cord palsy. It is of tremendous importance that the vagal electrode is a-traumatic and gives a stable signal. The exact parameters regarding "warning signs" have yet to be determined, but amplitude changes play a major role. Further studies are needed.

E-mail presenting author: dr.samvanslycke@gmail.com

Topic: Adrenal

Presentation type: Oral presentation

Gianluca Donatini ${ }^{1}$, Carlos Zerrweck ${ }^{2}$, Robert Caiazzo ${ }^{1}$, Ziad El-Kathib ${ }^{1}$, Thomas Gauthier ${ }^{1}$, Laurent Arnalsteen ${ }^{1}$, Bruno Carnaille ${ }^{1}$, Francois Pattou ${ }^{1}$

${ }^{1}$,France

${ }^{2}$ University of Lille, Mexico

\section{Long term survival after adrenalectomy for Adrenocortical Carcinoma. Case control study of Laparoscopic versus Open approach.}

Purpose: Laparoscopic adrenalectomy (LA) is the gold standard for benign adrenal lesions. The laparoscopic approach is increasingly accepted for adrenal metastases but remains controversial for adrenocortical carcinoma (ACC). We compared in a case-control study the outcome of LA versus open adrenalectomy $(\mathrm{OA})$ in the treatment of $\mathrm{ACC}$. Methods: Case-control study comparing the outcome of patients with ENSAT Stage I/II ACC and a size under $10 \mathrm{~cm}$ submitted to LA or OA in a referral center, from 1985 to 2011. Main outcomes analyzed were: peri-operative morbidity, overall survival, and disease free survival.
Results: Among 111 consecutive operations for ACC, 34 matched the inclusion criteria. LA and OA were performed respectively in 13 and 21 patients. Patient's characteristics were similar between groups. No conversion necessary in LA group. No difference in peri-operative morbidity. LA allowed an earlier discharge ( $p$

Conclusions: LA reported a shorter post-operative stay without jeopardizing the long-term oncological outcome in Stage I/II ACC $\leq 10 \mathrm{~cm}$. LA can be safely proposed for potentially malignant adrenal lesions $\leq 10 \mathrm{~cm}$, without evidence of extra-adrenal extension.

E-mail presenting author: giacko76@hotmail.com

Topic: Adrenal

Presentation type: Oral presentation

Anouk Scholten ${ }^{1}$, Robin M Cisco ${ }^{1}$, Menno R Vriens ${ }^{2}$, Wen T Shen ${ }^{1}$, Quan-Yang Duh ${ }^{1}$

${ }^{1}$ University of California, San Francisco, United States

${ }^{2}$ University Medical Center Utrecht, Netherlands

\section{Variant adrenal venous anatomy in 546 laparoscopic adrenalectomies}

Purpose: Identification and control of the adrenal vein is a critical step in laparoscopic adrenalectomy. Few prior studies describe variant adrenal venous anatomy and its relationship to tumor size, pathologic diagnosis, or operative outcomes.

Methods: We reviewed 546 consecutive laparoscopic adrenalectomies performed between 1993 and 2011 and compared tumor characteristics and outcomes of patients with variant anatomy to those with normal venous anatomy.

Results: Variant venous anatomy was encountered in 68 $(13 \%)$ adrenalectomies. Variants included no adrenal vein identified $(n=18)$, one central adrenal vein with additional small veins $(n=11)$, two $(n=20)$ or more than two $(n=14)$ adrenal veins, and variants of the adrenal vein with the hepatic or phrenic vein $(n=5)$. Variants occurred more often on the right than on the left ( $n=42,62 \%$ versus $n=26,38 \%)$. Compared to patients with normal anatomy, patients with variant anatomy had larger tumors (5.1 versus $3.3 \mathrm{~cm}, p<$ $0.001)$, more pheochromocytomas $(n=24,35 \%$ versus $n=$ $100,21 \%, p=0.016)$, and more blood loss (120 versus $66 \mathrm{~mL}, p=0.041)$. The rate of intra- and postoperative bleeding complications was similar between groups ( $p=$ 0.243 and $p=0.289$, respectively).

Conclusions: Understanding variants in adrenal venous anatomy is important to preventing excessive bleeding during laparoscopic adrenalectomy, particularly in patients with large tumors or pheochromocytoma.

E-mail presenting author: a.scholten-5@umcutrecht.nl 
Topic: Parathyroid

Presentation type: Oral presentation

Ralph Schneider ${ }^{1}$, Karin Stiasny-Kolster ${ }^{1}$, Detlef K. Bartsch $^{1}$, Katja Schlosser ${ }^{1}$

${ }^{1}$ Philipps University Marburg, Germany

The influence of parathyroidectomy on restless legs syndrome in patients with renal hyperparathyroidism

Purpose: Restless legs syndrome (RLS) is a common and poorly understood movement disorder that can cause significant sleep disruption. Although RLS is familial in about $50 \%$, secondary aetiologies such as iron deficiency and renal failure associated with hyperparathyroidism are common. The aim of this study was to analyze the influence of parathyroidectomy on RLS in patients with renal hyperparathyroidism. Methods: All patients who underwent parathyroidectomy between January and November 2011 were prospectively screened by a validated RLS-screening-questionnaire preoperatively and on the fifth postoperative day. Perioperative PTH and calcium-levels as well as the score of the questionnaire were analyzed.

Results: 21 patients (14 men, 7 women) with a mean age of $47.8 \pm 3.2$ years underwent parathyroidectomy. PTH-levels (normal-range 11-65 pg/ml) dropped perioperatively from $1171 \pm$ $120 \mathrm{pg} / \mathrm{ml}$ to $17 \pm 8 \mathrm{pg} / \mathrm{ml}$ and calcium-levels (normal range 2.2$2.7 \mathrm{mmol} / \mathrm{l})$ from $2.40 \pm 0.04 \mathrm{mmol} / 1$ to $1.95 \pm 0.04 \mathrm{mmol} / \mathrm{l}$. The score of the questionnaire (range 0-10) indicating a RLS significantly dropped from $6.10 \pm 0.5$ to $4.29 \pm 0.6(p=0.0038)$.

Conclusions: Parathyroidectomy seems to have a significant influence on the severity of RLS. The restoration of an adequate calcium- and mineral-balance as indicated by the decrease of PTH-levels may be one reason. However, large prospective trials are required to analyze this observation in patients undergoing parathyroidectomy for renal hyperparathyroidism.

E-mail presenting author: ralph.schneider@med.uni-marburg.de

Topic: Thyroid

Presentation type: Oral presentation

Marco Raffaelli ${ }^{1}$, Carmela De Crea ${ }^{1}$, Luca Sessa ${ }^{1}$, Piero Giustacchini ${ }^{1}$, Rocco Bellantone ${ }^{1}$, Celestino Pio Lombardi ${ }^{1}$

${ }^{1}$ Università Cattolica del Sacro Cuore, Italy

\section{Can intraoperative frozen section influence the extension of central neck dissection in cN0 papillary thyroid carcinoma?}

Purpose: Ipsilateral central compartment node dissection (CCD) has been proposed to reduce the morbidity of prophylactic bilateral CCD in papillary thyroid carcinoma (PTC), despite bilateral metastases are found in about $25 \%$ of patients. We aimed to verify if frozen section examination (FSE) can identify patients who could benefit from bilateral CCD.
Methods: All the consenting patients with clinically unifocal PTC, without any preoperative evidence of lymph node involvement, treated between September 2010 and September 2011 underwent total thyroidectomy plus bilateral CCD. Ipsilateral central compartment nodes were sent for FSE. Results: Forty-eight patients were included. Mean number of removed nodes was $12.8 \pm 6.8$. Final histology showed lymph node metastases in 20 patients: ipsilateral in 14, bilateral in 6 . FSE accurately predicted lymph node status in 43 patients ( 28 node negative, 15 node positive). Five node metastases were not detected at FSE: 3 were micrometastases $(<2 \mathrm{~mm})$. Sensitivity, specificity and overall accuracy of FSE in definition of $\mathrm{N}$ status were $75 \%, 100 \%$ and $89 \%$, respectively.

Conclusions: FSE is accurate in predicting node metastases in clinically unifocal N0 PTC and can be useful in determining the extension of CCD. False negative results are reported mainly in case of micrometastases, which usually have limited clinical implications.

E-mail presenting author: marcoraffaelli@rm.unicatt.it

Topic: Thyroid

Presentation type: Oral presentation

Parameswaran Rajeev ${ }^{1}$, Sohail Ahmed ${ }^{2}$, Tarek Ezzat ${ }^{2}$, Gregory Sadler ${ }^{2}$, Radu Mihai ${ }^{2}$

${ }^{1}$ Oxford University Hospitals NHS Trust

${ }^{2}$ Oxford University Hospitals, United Kingdom

Does the number of positive lymph nodes in the central compartment have any prognostic impact in papillary thyroid cancer?

Purpose: Central compartment lymph node (CCLN) metastasis in papillary thyroid cancer (PTC) is associated with higher risk of locoregional recurrence and distant metastasis. This study evaluated the prognostic implication of the number of metastatic CCLN in PTC.

Methods: Prospective data collection on 91 patients who underwent total thyroidectomy and CCLN dissection with or without lateral neck dissection due to PTC between Jan2005-Dec2010. Number of positive CCLN was correlated with known prognostic factors (age, gender, tumour size, extrathyroidal extension, and lateral node metastasis).

Results: Patients were divided into three groups according to the number of positive CCLN: group $A=0(n=35) ; B=1-2$ $(n=32)$ and $\mathrm{C}=>3(n=24)$. The risk of lateral compartment disease increased in parallel with the number of positive CCLN (31\% vs $50 \%$ vs $75 \%$ in groups A-B-C respectively; $p<0.004)$. Gender/age/tumour size/extrathyroidal extension did not correlate with number of positive CCLN. At 1-yr follow-up the mean $\mathrm{Tg}$ values increased with number of nodes positive (12.3 vs 42.3 vs 91.48$)$ but with no significant difference in RAI* uptake (1.25 vs 1.14 vs 2.63 ). 
Conclusions: The number of CLN metastasis is a risk factor for lateral compartment disease with no impact on any other prognostic markers.

E-mail presenting author: malumols@live.co.uk

Topic: Thyroid

\section{Presentation type: Oral presentation}

Jose Manuel Rodriguez Gonzalez ${ }^{1}$, Antonio Rios Zambudio ${ }^{1}$, Angela Cepedo ${ }^{1}$, Maria D. Balsalobre Salmeron ${ }^{1}$, Nuria M. Torregrosa Perez ${ }^{1}$, Manuel Reus ${ }^{1}$, Pascual Parrilla Paricio ${ }^{1}$

${ }^{1}$ Hospital U. Virgen de la Arrixaca, Spain

\section{Elastography in the thyroid nodule diagnosis. prospective study}

Purpose: There is generally a considerable difference between the rigidity of normal thyroid tissue and malignant nodules. The objective of this study is to analyze the differences between benign and malignant thyroid nodules.

Methods: Study: Prospective. Selection Criteria: Unique nodules or multinodular goiters with a dominant nodule. Study design: We used an ultrasound, with an elastograph, a B mode ultrasound and Color Doppler. The elastography was classified into 5 groups.

Results: 178 patients were analyzed; 40 were malignant according to histology. The elastograph diagnosis score was $4-5$ in $40(23 \%), 3$ in $34(19 \%)$ and $1-2$ in $104(58 \%)$.

All the patients with 5 in the elastograph diagnosis score were malignant. All the patients except four, with elastograph 4 were malignant. In three patients with elastograph score 4-5, cytology was benign and the histology revealed malignancy.

Of the patients with the elastograph 3 , in four cases (12\%) the diagnosis was malignancy. All the nodules with elastograph 1-2, were benign.

Conclusions: Elastograph score 4-5 is a strong predictor of malignancy. Elastograph score 1-2 is a strong predictor of benignity. The elastography could be useful as a diagnostic technique for ruling out malignancy in the thyroid nodule.

E-mail presenting author: jmrodri@um.es

Topic: Thyroid

Presentation type: Oral presentation

Maya Belitova ${ }^{1}$, Rumen Pandev ${ }^{1}$, Dimitar Karadimov ${ }^{1}$, Ahmad Kouzi ${ }^{1}$

${ }^{1}$ University Hospital "Queen Giovanna", Bulgaria

\section{Fast Track One-Day Thyroid Surgery—are we in the right to omit post-anesthesia care unit?}

Purpose: The objectives were to determine if patients, who passed our Fast Track Protocol could be safely discharged from the operating room to the post-surgical area after OneDay thyroid surgery. Cost-effectiveness of this approach was also analyzed.

Methods: 516 patients operated under general anesthesia were retrospectively analyzed. Surgery included: total and near-total thyroidectomies in 104 patients, hemithyroidectomy in 286 , subtotal bilateral and partial thyroidectomies in 31 , completion thyroidectomies in 95 cases. 275 from the operations $(53,3 \%)$ were carried out as minimally invasive procedures. Our Fast-Track protocol consists of three consecutive parts: surgical, anesthetic and patient's readiness assessment. The last part was scored (0-1-2) by seven criteria: level of consciousness, physical activity, hemodynamic stability, respiratory stability, oxygen saturation, postoperative pain, emetic status.

Results: Patients who met all parts of our Fast-Track protocol bypassed the postanesthesia care unit (PACU). None of them was readmitted. The major complications were hemorrhage $(0,3 \%)$ and transient nerve palsy $(1,4 \%)$.

Conclusion: A minimal score of 12 (with no score? 1 in any individual category) would be required to bypass the PACU. The cost-savings were $>35 \%$ in comparison with the current one-day thyroid surgery approach. Fast-Track protocol saves money but is unable to predict the occurrence of major surgical complications.

E-mail presenting author: belitova@mail.bg

Topic: Parathyroid

Presentation type: Oral presentation

Pier Francesco Alesina ${ }^{1}$, Martin K Walz ${ }^{2}$

${ }^{1}$ Kliniken Essen-Mitte

${ }^{2}$ Kliniken Essen-Mitte, Department Surgery, Germany

\section{Feasibility of video-assisted bilateral neck exploration for patients with primary hyperparathyroidism.}

Purpose: Video-assisted parathyroidectomy (MIVAP) is generally adopted for patients affected by primary hyperparathyroidism (pHPT) with clear preoperative localization. Standard bilateral neck exploration (BNE) is considered the obligate operation for patients with unlocalized glands. We reviewed our experience of minimally invasive video-assisted BNE in patients with pHPT and negative or discordant localization studies.

Methods: From a prospective series of 548 minimally invasive video-assisted parathyroidectomies for $\mathrm{pHPT}, 95$ patients (17 male, 78 female; mean age: 58 years) who had failed localization studies underwent BNE using the video-assisted technique. Operative time, complications, conversions to standard cervical exploration and cure rate were analyzed.

Results: MIVAP with BNE was successfully completed in $90(95 \%)$ patients with 5 conversions. Mean operative time 
was $56 \pm 34$ min (range: $20 €$ “180 min). Recurrent laryngeal nerve palsy occurred in one patient. Biochemical cure was achieved in 93 patients $(98 \%)$, in 89 patients after the first operation, in 4 patients by video-assisted $(n=2)$ or conventional $(n=2)$ re-exploration. One patient remained with persistent disease even after repeated open BNE; a second one developed recurrence disease 3 years after the first exploration.

Conclusion: In experienced hands, video-assisted BNE for pHPT is feasible, safe and gives results equivalent to the conventional open technique.

E-mail presenting author: pieroalesina@libero.it

Topic: Parathyroid

Presentation type: Oral presentation

Philipp Riss ${ }^{1}$, Asari Reza ${ }^{1}$, Christian Scheuba ${ }^{1}$, Bruno Niederle $^{1}$

${ }^{1}$ Medical University of Vienna, Austria

\section{Current trends in surgery for renal hyperparathyroidism (RHPT) - an international survey}

Purpose: Indications and results of cyropreservation and replantation of cryopreserved parathyroid tissue and the application of intraoperative PTH-monitoring are not well documented in RHPT The current trends in surgery for RHPT should be evaluated in an international online survey. Methods: 33 questions regarding parathyroid surgery, surgical management of RHPT, parathyroid cryopreservation and PTH-monitoring were sent to members of various societies of endocrine surgeons.

Results: 86 surveys were analyzed. $61.6 \%$ reported more than 50 parathyroid surgeries per year. $62.7 \%$ operated on less than 16 patients with RHPT per year. Total (with/without autotransplantation) or subtotal parathyroidectomy was the standard procedure in $98.8 \% .40 .7 \%$ performed immediate autotransplantation. In most cases the onset of graft function was documented later than 1 week after autotransplantation. Cryopreservation was routinely performed in $27.4 \% 10.7 \%$ performed replantation in more than 5 patients (hypo- or aparathyroidism: $n=41$; fresh graft failure: $n=13$; reoperations: $n=9.46 .2 \%$ used routinely intraoperative PTHmonitoring in RHPT. Its influence on surgical strategy was stated in $40 \%$.

Conclusions: Cryopreservation is only performed in $27.4 \%$ routinely to avoid hypoparathyroidism in selected patients. Intraoperative PTH-monitoring has a surprisingly high acceptance with criteria similar to primary hyperparathyroidism. The survey reflects the divergent strategies for autotransplantation, cryopreservation and PTH-monitoring.

E-mail presenting author: philipp.riss@meduniwien.ac.at
Topic: Adrenal

Presentation type: Oral presentation

Guillaume muller ${ }^{1}$, Fabien saint ${ }^{2}$, Antoine hamy ${ }^{3}$, Jeanchristophe lifante ${ }^{4}$, Bruno carnaille ${ }^{5}$, Frederic sebag ${ }^{6}$, Fabrice menegaux $^{7}$, Jean-francois henry ${ }^{6}$, Eric mirallie ${ }^{1}$

${ }^{1}$ Chu nantes

${ }^{2} \mathrm{Chu}$ amiens

${ }^{3} \mathrm{Chu}$ angers

${ }^{4}$ Ch lyon sud

${ }^{5}$ Hopital claude huriez lille

${ }^{6}$ Hopital la timone marseille

${ }^{7}$ Hopital la pitie-salpetriere Paris, France

Pheochromocytoma revealed by acute heart failure. A multicenter study about morbidity and mortality of surgery

Purpose: Acute heart failure (AHF) secondary to catecholamine over production rarely reveals a pheochromocytoma. The aim of this study was to evaluate the management of patients with pheochromocytoma diagnosed at the time of an AHF.

Methods: Data of 12 patients were retrospectively reviewed. The diagnosis of AHF was defined by the decrease of left ventricular ejection fraction or the use of a circulatory assist. They had adrenalectomy in emergency surgery or later. Morbidity and mortality of surgery was studied.

Results: Adrenalectomy was performed in emergency for 4 patients (33\%) and for the 8 other patients $(67 \%)$ with a median delay of 37 days (7-180). 8 patients had circulatory assist (67\%). 5 of them had a circulatory assist and a delayed surgery (42\%), 2 of them had a circulatory assist followed by emergency surgery and 1 had emergency surgery immediately followed by circulatory assist. Emergency surgery had perioperative complications: 1 circulatory arrest, 2 major bleedings, 1 intestinal ischemia, 1 hémoperitoneum. 1 patient died at day 5. Postoperative course of patients with delayed surgery was uneventful. Conclusions: The AHF revealing a pheochromocytoma is a rare and serious event. Patients with emergency surgery have more complications than those with delayed surgery.

E-mail presenting author: guillaumuller@gmail.com

Topic: Thyroid

Presentation type: Oral presentation

Aleksander Konturek ${ }^{1}$, Marcin Barczynski ${ }^{2}$, Wojciech Wierzchowski ${ }^{3}$, Malgorzata Stopa ${ }^{4}$, Wojciech Nowak $^{4}$

${ }^{1} 3$ rd Chair of General Surgery Jagg Univ

${ }^{2}$ IIIrd Chair Gen. Sur Jagg Univ

${ }^{3}$ Chair Of Pathology Jagg Univ.

${ }^{4}$ IIrd Chair Gen. Sur Jagg Univ, Poland

\section{Coexistence of papillary thyroid cancer with Hashimoto} thyroiditis.

Purpose: Conflicting data have been reported with regard to the Hashimoto thyroiditis (HT) and risk of malignancy. The 
aim of this study was to evaluate coexistence of papillary thyroid cancer (PTC) with HT.

Methods: A retrospective cohort study. HT was diagnosed in 452 (F:M ratio $=405: 47$, median age $53.5 \pm 12.1$ years) of 7545 patients qualified for thyroidectomy throughout the years 2002 and 2010. Pathological reports were reviewed to identify prevalence of PTC in HT versus non-HT patients. Results: PTC was diagnosed in 106 of 452 (23.5\%) HT patients versus 530 of 7093 (7.5\%) non-HT patients $(p<$ $0.001)$. Metastases to level VI lymph nodes were observed in 81 of 106 (76.4\%) patients with PTC in HT versus 121 of $530(22.8 \%)$ patients with PTC in non-HT disease $(p<0.001)$. Conclusions: HT was associated with three-fold increased prevalence of PTC than other non-HT thyroid diseases and spread of PTC to level VI lymph nodes was four-fold more frequent in HT than in non-HT patients.

E-mail presenting author: okont@mp.pl

Topic: Adrenal

Presentation type: Oral presentation

Vasilis Constantinides ${ }^{1}$, Ioannis Christakis ${ }^{1}$, Touska Philip ${ }^{1}$, Fausto Palazzo ${ }^{1}$

${ }^{1}$ Imperial College NHS Trust, United Kingdom

\section{Retroperitoneoscopic versus laparoscopic adrenalectomy —a meta-analysis}

Purpose: Laparoscopic adrenalectomy (LA) is the gold standard approach to most non malignant adrenal tumours. Retroperitoneoscopic adrenalectomy (RA) is an increasingly popular alternative to this approach but the potential benefits derived from this approach have not been clearly defined.

Methods: A literature search was performed for all comparative studies between RA and LA. Meta-analysis was performed according to PRISMA guidelines. Odds ratios (OR) and standardised mean differences (SMD) were used to compare dichotomous and continuous outcomes respectively.

Results: Twenty studies were included reporting on 873 LA and 573 RA. Length of hospital stay (LOS) was significantly shorter in the RA group (SMD-0.77,95\% CI -1.29-$0.25)$. On subgroup analysis only posterior RA was associated with reduced LOS (SMD $-1.45,95 \%$ CI -CI -2.76-$0.14)$ when compared to LA. There was no difference in operative time, blood loss, time to ambulation and oral intake or complication rates between techniques. No difference was found in any of the outcomes for studies matched for tumour size, body mass index or published after 2005 .

Conclusions: RA overall has equivalent outcomes to LA but is associated with a reduced LOS. A large-scale randomised controlled trial is necessary to provide definitive conclusions.

E-mail presenting author: vascons@doctors.org.uk
Topic: GI neuroendocrine

Presentation type: Oral presentation

Felix Watzka ${ }^{1}$, Christiane Laumen ${ }^{1}$, Christian Fottner ${ }^{1}$, Matthias Weber ${ }^{1}$, Hauke Lang ${ }^{1}$, Thomas Musholt ${ }^{1}$

${ }^{1}$ university medical center of Mainz, Germany

\section{Resection strategies for neuroendocrine pancreatic tumors}

Purpose: Due to its rarity and lack of prospective trials, the optimal treatment of PNETs is still debated. Recommendations gathered by retrospective analyses of patient data should be based on the new classification of neuroendocrine neoplasms.

Methods: In a retrospective single-centre study (1990 to 2011), 127 PNETs patients were analyzed. Tumor stage and type of resections were analyzed to evaluate successful treatment strategies.

Results: 79 patients (62\%) were diagnosed with stage I or II, 48 patients (38\%) with stage III or IV disease. $42.3 \%$ of all PNETs were non-functional. Surgical interventions consisted of 53 enucleations and 25 distal resections in patients with stage I or II disease. 53 patients with stage III or IV disease received a distal resection (22) or partial duodenopancreatectomy (13). Exploration without resection was performed in 8 patients. Stage-dependent 10-year survival rates were $91.0 \%$ (stage I and II, $n=79$ ) and $37.5 \%$ (stage III and IV, $n=48$ ). Conclusion: PNETs have a good prognosis, if they are welldifferentiated and resected completely. Organ-preserving resection does not impair the prognosis of tumors of stage I or II. In case of hepatic metastasis and advanced tumor stage, surgical reduction can reduce symptoms and improve the survival.

E-mail presenting author: felix.w1@gmx.de

Topic: Thyroid

Presentation type: Oral presentation

Gabriele Materazzi ${ }^{1}$, Ayman Mismar $^{2}$, Marco Biricotti ${ }^{3}$, Alessandra Fosso ${ }^{3}$, Rocco Rago ${ }^{3}$, Paolo Miccoli ${ }^{3}$

${ }^{1}$ University, Italy

${ }^{2}$ University of Jordan, Jordan

${ }^{3}$ University of Pisa, Italy

Efficacy on pain of Pubivacaine versus Ropivacaine infiltration before thyroidectomy: prospective randomized study.

Purpose: Control of pain after thyroidectomy is very important. The efficacy of wound infiltration with local analgesics are not settled. We run a prospective double blinded study comparing Pubivacaine $0.5 \%$ versus Ropivacaine $0.75 \%$ efficacy in reducing pain after thyroidectomy.

Methods: Inclusion criteria were: age 18-65, thyroid volume lower than $90 \mathrm{ml}$, benign disease. Exclusion criteria were: redo surgery, malignancy, operative time longer than $90 \mathrm{~min}$, incision longer than $10 \mathrm{~cm} 60$ patients were randomly divided into Group A: control group (no wound infiltration); group B: infiltration of $10 \mathrm{ml}$ Pubivacaine $0.5 \%$, group C: infiltration 
of $10 \mathrm{ml}$ Ropivacaine $0.75 \%$. Pain was assessed by Visual Analogue Scale after 1,4,8,16 h postoperatively. Surgery was performed by the same surgeon with standard technique. Local infiltration was done immediately before incision. All patients received the same analgesic therapy postoperatively.

Results: Ropivacaine group showed a statistically significant decrease in pain perception at $1 \mathrm{~h}$ postoperatively. After $4 \mathrm{~h}$ of operation neither Ropivacaine nor Pubivacaine showed an effect on pain perception.

Conclusion: Ropivacaine proved to significantly decrease pain perception within $1 \mathrm{~h}$ of operation, then we suggest the use of this drug to improve immediate postoperative recovery after thyroidectomy.

E-mail presenting author: gmaterazzi@yahoo.com

\section{Best Poster (with short oral presentation)}

Topic: Parathyroid

Presentation type: Short oral presentation

Bojan Kovacevic ${ }^{1}$, Mile Ignjatovic ${ }^{1}$, Vladimir $\mathrm{Cuk}^{1}$, Vladan Zivaljevic $^{2}$, Scepanovic Milena ${ }^{1}$, Ivan Paunovic ${ }^{1}$

${ }^{1}$ Zvezdara University Clinical Centre

${ }^{2}$ Clinical Center of Serbia, Serbia

Early prediction of persistent or recurrent secondary hyperparathyroidism after parathyroidectomy and autotransplantation

Purpose: Persistent and recurrent hyperparathyroidism are still the most common complications of parathyroidectomy for secondary hyperparathyroidism. Intraoperative identification of valid predictive factors for recurrent disease may have important influence on surgical strategy and significantly reduce percent of patient with persistent or recurrent disease. Methods: This study included forty-three consecutive patients who underwent parathyroidectomy for a severe form of secondary hyperparathyroidism that is unresponsive to medical treatment. The serum parathyroid hormone, calcium, and phosphorus levels were measured prior to surgery, every morning after surgery for 5 days, and on the first, sixth, and eighth postoperative months.

Results: Intraoperative decline of the parathyroid hormone for more than $91.6 \%$ from preoperative values, $15 \mathrm{~min}$ after excision of the last gland, showed $100 \%$ sensitivity and $86 \%$ specificity in prediction of persistent hyperparathyroidism. We did not confirm predictive value of any other biochemical parameters that were measured in this study.

Conclusions: Intraoperative monitoring of the parathyroid hormone decline during parathyroidectomy for secondary hyperparathyroidism can reliably identify patients with unsuccessful operation. The application of this technique during parathyroidectomy could have significant impact on surgical strategy and increase percent of successfully treated patients.

E-mail presenting author: bkzvez@gmail.com
Topic: Thyroid

Presentation type: Short oral presentation

Arturs Ozolins ${ }^{1}$, Zenons Narbuts ${ }^{1}$, Ilze Strumfa ${ }^{1}$, Guna Volanska $^{2}$, Kaspars Stepanovs ${ }^{2}$, Janis Gardovskis ${ }^{1}$

${ }^{1}$ Riga Stradins University

${ }^{2}$ Pauls Stradins University Hospital, Latvia

Immunocytochemical panel as an adjunct to fine-needle aspiration of the thyroid in various thyroid pathologies

Purpose: Fine needle aspiration (FNA) with cytologic evaluation is the most reliable tool for distinction between benign and malignant thyroid tumors, but cytologic diagnosis remains undetermined in $20 \%$ of cases. The study was performed to investigated the diagnostic potential of a set of three molecular markers in preoperative evaluation of different thyroid tumors. Methods: Sixty-eight thyroid FNA cases confirmed by subsequent surgical resection specimens were selected. Immunocytochemistry for HBME-1, CD56 and E-Cadherin was performed. The study group consisted of 25 papillary carcinomas (PC) and 1 follicular carcinoma (FC) as well as 22 follicular adenomas (FA) and 20 cases of colloid goiter (CG). Results: Notable HBME-1 expression was found in PC 24/ 25 (96\%), none of benign lesions. E-Cadherin and CD56 expression was significantly weakened in PC but enhanced in FA 16/22 (72.7\%) and 12/22 (54.5\%) respectively. In case of CG markers expression was not reaching $10 \%$.

Conclusions: Immunocytochemistry is of value as an ancillary test to enhance the diagnostic accuracy of thyroid FNA biopsies. The expression profiles of three markers (HBME-1, E-Cadherin and CD56) served as adjunct to standard cytomorphology criteria to enhance its diagnostic accuracy.

E-mail presenting author: archa_oz@inbox.lv

Topic: Parathyroid

Presentation type: Short oral presentation

Monique Ohe ${ }^{1}$, Rodrigo Santos ${ }^{2}$, Murilo Neves ${ }^{2}$, Aluizio Carvalho $^{3}$, Ilda Kunii ${ }^{4}$, Marcio Abrahao $^{2}$, Onivaldo Cervantes $^{2}$, Marise Lazaretti-Castro ${ }^{4}$, Jose Gilberto Vieira ${ }^{4}$ ${ }^{1}$ UNIFESP/EPM

${ }^{2}$ Head and Neck Surgery UNIFESP/EPM

${ }^{3}$ Nephrology UNIFESP/EPM

${ }^{4}$ Endocrinology and Metabolism UNIFESP/EPM, Brazil

Tissue selection with or without stereomicroscopy in surgical treatment for renal hyperparathyroidism

Purpose: The best surgical approach for renal hyperparathyroidism is yet to be defined, since neither high recurrence rates nor risks of definitive hypoparathyroidism are intended.

Methods: 123 patients underwent total parathyroidectomy with presternal intramuscular autotransplantation, being 
divided in: G1: 66 patients operated 04/2000-04/2005 with tissue selection based on macroscopic findings; G2: 57 patients operated 03/2008-10/2009 with tissue selection by resorting to Leica StereoZoom-S8APO Stereomicroscope. Intraoperative-PTH (Elecsys-Roche) measured in 100 patients $(81.3 \%)$. Data presented as average.

Results: G1: 38 haemodialysis patients, 28 renal-graft, $38 \mathrm{Fe}-$ male/28Male, aged 40.8 (14-62); intact parathormone (iPTH) $73.5 \mathrm{pg} / \mathrm{mL}, 82 \mathrm{pg} / \mathrm{mL}$ and $80 \mathrm{pg} / \mathrm{mL} \mathrm{12,} \mathrm{24,}$ 36 months post-operative respectively in dialysis patients, and $54.9 \mathrm{pg} / \mathrm{mL}, 61.7 \mathrm{pg} / \mathrm{mL}$ and $70.5 \mathrm{pg} / \mathrm{mL}$ in renal-graft patients. Hypoparathyroidism was observed in $4(6.06 \%)$ and graft recurrence in 6, all in dialysis patients. G2: 32 dialysis patients, 25 renal-graft, $25 \mathrm{Female} / 32 \mathrm{Male}$, aged 48.4 (26-74); iPTH $58.1 \mathrm{pg} / \mathrm{mL}$ and $62.8 \mathrm{pg} / \mathrm{mL} 12$ and 18 months post-operative respectively in dialysis patients, and $57.4 \mathrm{pg} / \mathrm{mL}$ and $61.7 \mathrm{pg} / \mathrm{mL}$ in renal-graft patients. All patients were cured except 1, from dialysis group, who presented graft-dependent recurrence 6 months post-operative, and 1 renal-graft patient with definitive hypoparathyroidism.

Conclusion: Stereomicroscopy in tissue selection was helpful in obtaining low incidence of hypoparathyroidism and graft-dependent recurrence.

E-mail presenting author: moniqueohe@uol.com.br

Topic: Thyroid

Presentation type: Short oral presentation

Gregorio Scerrino ${ }^{1}$, Nunzia Cinzia Paladino ${ }^{1}$, Angela Inviati $^{1}$, Valentina Di Paola ${ }^{1}$, Silvia Di Giovanni ${ }^{1}$, Roberto Gullo $^{1}$, Francesco Cupido ${ }^{2}$, Piero Luigi Almasio ${ }^{1}$, Sebastiano Bonventre ${ }^{1}$

${ }^{1}$ University of Palermo

${ }^{2}$ Univerity of Palermo, Italy

\section{Esophageal motility changes after thyroidectomy: possible associations with postoperative voice and swallowing disorders}

Purpose: Swallowing and voice impairment are common after thyroidectomy. Several studies investigated these symptoms by means of interviews or questionnaires. We evaluated short term functional changes of oesophageal motility in a series of patients underwent total thyroidectomy and their associations with these disorders.

Methods: 36 consenting patients were prospectively recruited. Eligibility criteria: thyroid volume $\leq 60 \mathrm{ml}$, benign disease, age between 18 and 65 years. Exclusion criteria: previous neck surgery, severe thyroiditis, hyperthyroidism, pre- or postoperative vocal cord palsy. Voice Impairment Score (VIS), Swallowing Impairment Score (SIS), LES pressure, esophageal motility, UES pressure and coordination were evaluated preoperatively and $30-45$ days postoperatively.

Results: Postoperative swallowing impairment (appearance or worsening of dysphagia) was found in $20 \%$ of patients. Voice impairment in $30 \%$. Both preoperative and postoperative esophageal motility were similar. All patient showed an average decrease of $25 \%$ of UES pressures, even though it was within normal range. Swallowing alterations were associated with UES incoordination (Conclusions: After uncomplicated thyroidectomy, decreased UES pressure could explain both pharyngeal (dysphagia) and laryngeal (vocal impairment) acid exposure. Perspectively, PPI therapy protocols should be evaluated.

E-mail presenting author: gregorio.scerrino@tiscali.it

Topic: Thyroid

Presentation type: Short oral presentation

Gyan Chand ${ }^{1}$, Sudhi Agarwal ${ }^{2}$, Anjali Mishra ${ }^{2}$, Gaurav Agarwal $^{2}$, Amit Agarwal ${ }^{2}$, A.K. Verma ${ }^{2}$, S.K. Mishra ${ }^{2}$

${ }^{1}$ Sanajay Gandhi Post Graduate Institute o

${ }^{2}$ SGPGIMS, India

\section{Correlation of histological subtypes of papillary thyroid} carcinoma with clinical outcome: A single centre experience.

Purpose: Papillary thyroid cancer is the most common thyroid cancer with good overall survival. Histologic subtypes, may play a role in varied clinical presentation and prognosis.

The aim of the present study was to investigate the corelation of papillary thyroid cancer subtypes with their clinical behaviour and prognosis.

Methods: Retrospective analysis of cases with confirmed PTC, who underwent total thyroidectomy as primary treatment from 1997 till 2009 at our institute.

Results: Total 194 cases. Classical Variants (CV)(78.4\%), Follicular Variants (FV)(9.3\%), Poorly differentiated Variants (PDV)(4.6\%), Tall cell Variants (TCV)(3.6\%), Oncocytic Variants (OV) (1.5\%), and not available2.5\%. PDV present at older age, all subtypes were common in females, mean tumour size was large in PDV. Lymph node metastasis was high in OV \& CV but the extrathyroidal invasion was common in PDV. Multicentricity was common in TCV and bilaterality was common in $\mathrm{n} \mathrm{OV}$. The follow-up ranges from 02 to 218 months; recurrence was commonest in $\operatorname{PDV}(33.3 \%$ vs CV 13.2\%) and Overall survival was lowest in PDV (19.4 month vs CV 27.9 months).

Conclusion: The poorly differentiated subtypes of PTC present in older age, aggressive and have poor survival than the other variants including the tall cell subtypes. Follicular subtypes have relatively better outcome.

E-mail presenting author: drgyanchandpgi@gmail.com 
Topic: Thyroid

Presentation type: Short oral presentation

Dimitrios Linos ${ }^{1}$, Andreas Kiriakopoulos ${ }^{2}$, Eleni Linos ${ }^{3}$, Athanassios Petralias ${ }^{4}$

${ }^{1}$ Hygeia Hospital, Athens, Greece

${ }^{2}$ Hygeia Hospital, Greece

${ }^{3}$ University of California San Francisco, United States

${ }^{4}$ Athens University of Economics and Bus., Greece

\section{The effect of scar perceptions after thyroid and parathyroid surgery on patients' physical and mental health}

Purpose: There is lack of rigorously validated evidence on how the scar appearance and self-consciousness as perceived by patients following thyroidectomy or parathyroidectomy surgery, affect their physical and mental health. Methods: We analysed a random sample of 696 patients who underwent thyroidectomy or parathyroidectomy between January 2000 and March 2010. Patients' perception with respect to their scars was assessed using the validated $\mathrm{Pa}$ tient Scar Assessment Questionnaire (PSAQ), while their physical and mental health was measured using the SF-36 health survey questionnaire. After performing validity and reliability analysis, we estimated the effect of scar appearance and self-consciousness PSAQ subscales to the patients' physical and mental health, controlling for 12 sociodemographic and surgical characteristics.

Results: The PSAQ appearance and consciousness subscale scores are significantly correlated $(p<0.005)$ with patients' mental health. Lower patients' self-consciousness regarding their scar, is significantly related $(p<0.005)$ to worse physical and mental health. This result holds true irrespectively of the surgical procedure used (conventional or minimal invasive), the histological diagnosis (benign or malignant disease), or the presence of postoperative complications.

Conclusions: Self-consciousness regarding the scar might lead to worse physical and mental health, irrespectively of the length of the scar from a minimally invasive or not thyroidectomy or parathyroidectomy.

E-mail presenting author: dlinos@hms.harvard.edu

Topic: Thyroid

Presentation type: Short oral presentation

Johannes Järhult ${ }^{1}$, Kalle Landerholm ${ }^{1}$

${ }^{1}$ Länssjukhuset Ryhov, Jönköping, Sweden

\section{Surgery for retrosternal goitre}

Purpose: Patients with goitre descending below the plane of the thoracic inlet are usually recommended surgery although this recommendation is not based on much scientific evidence. This study was carried out to see whether the complication rates after these operations differ from those after ordinary neck surgery.

Methods: 107 women and 41 men with retrosternal goitre were operated in the District Hospital at Eksjö, Sweden, between 1984 and 2011. Median age was 63 years (17-84). Only 40 of them $(27 \%)$ complained of compressive symptoms. All except 3 goitres were removed via a standard collar incision.

Results: Reoperation due to bleeding occurred in 3 patients $(2,0 \%) .13$ had palsy of one recurrent laryngeal nerve; 7 of them $(4,7 \%)$ became permanent. Another 2 patients $(1,4 \%)$ had permanent hypoparathyroidism. 3 old patients died postoperatively.-The corresponding complication frequencies in 549 patients with non-thoracic thyroid surgery at the same institution were 2,0 and $1,8 \%$ for reoperations and permanent nerve palsy, respectively, and $1,6 \%$ for permanent hypoparathyroidism.

Conclusions: Transcervical removal of retrosternal goitres may lead to a high risk for permanent palsy of the recurrent laryngeal nerve. Surgery for retrosternal goitre, particularly in old people without compressive symptoms, needs to be evaluated in randomized studies.

E-mail presenting author: johannes.jarhult@lj.se

Topic: Thyroid

Presentation type: Short oral presentation

M. Bossotti ${ }^{1}$, A. Borasi1, A. Barbersis ${ }^{1}$, P. De Paolis ${ }^{1}$, F. Botto $\mathrm{Micca}^{2}$, R. Parente ${ }^{2}$, S. Corvisieri ${ }^{3}$, I. Messuti ${ }^{3}$, F. Orlandi ${ }^{3}$

${ }^{1}$ Department of General Surgery, Presidio Sanitario Gradenigo, Turin, Italy

${ }^{2}$ Department of Histopathology, Presidio Sanitario Gradenigo, University of Turin, Turin, Italy

${ }^{3}$ Department of Internal Medicine, Section of Endocrinology, Presidio Sanitario Gradenigo, University of Turin, Turin, Italy

\section{Immunohistochemistry in surgical selection of follicular thyroid nodules with indeterminate cytology}

Purpose: Fine-needle aspiration biopsy (FNAB) has a central role in the diagnosis of thyroid nodules. An important limitation of FNAB is the lack of sensitivity in the evaluation of "follicular lesions". Immunohistochemistry could improve the diagnostic accuracy, enabling a better selection of patients requiring surgery versus follow-up and reducing the number of unnecessary thyroidectomies for benign lesions.

Methods: From March 2008 to April 20111342 thyroid FNABs were carried out in our Endocrinology Unit. The diagnoses of "follicular lesions" (THY3) were 63 (4.7\%). We evaluated 38 of these "follicular lesions", looking for 
immunohistochemistry expression of Galectin-3, HBME-1 and CK-19. Out of these 38 THY3, 14 (36.8\%) resulted carcinoma at histology, 1 UMPT, 5 adenomas and 18 benign lesions.

Results: The concordant panel between two markers resulted positive in 10 cases ( 4 benign and 6 malignant) and negative in 8 cases ( 6 benign and 2 malignant), with a sensibility of $75 \%$ and specificity of $60 \%$.
Conclusions: In agreement with the literature, our results suggest that the application of this antibodies' panel in THY3 lesions may improve the accuracy of morphologic evaluation and may optimize the management of patients, in terms of surgery versus follow-up, type of surgery and priority inherent waiting lists.

E-mail presenting author: bossotti.maurizio@tiscali.it 


\section{Author index}

$\begin{array}{ll}\text { Alesina P F 851 } & \text { Kovacevic B 854 } \\ \text { Belitova M 851 } & \text { Linos D 843, 856 } \\ \text { Bossotti M } 856 & \text { Lorenz K 843, 846 } \\ \text { Chand G 855 } & \text { Lu M 845 } \\ \text { Constantinides V 853 } & \text { Makay O 844 } \\ \text { Donatini G } 849 & \text { Markogiannakis H } 847 \\ \text { Dzodic R } 847 & \text { Materazzi G 853 } \\ \text { Gonzalez J M R 851 } & \text { Muller G 852 } \\ \text { Gonzalez-Sanchez C 844 } & \text { Nordenström E 848 } \\ \text { Iacobone M 843, 845 } & \text { Ohe M 854 } \\ \text { Järhult J 856 } & \text { Ozolins A 854 } \\ \text { Konturek A } 852 & \text { Perez N M 848, 851 }\end{array}$

Raffaelli M 843, 850

Rajeev P 850

Riss P 852

Rolighed L 845

Salido S 846

Scerrino G 855

Schneider R 850

Scholten A 849

Sitges-Serra A 843, 844

Van Slycke S 843, 848

Watzka F 853

Wirowski D 847 